\title{
INFORMACIÓN SOBRE SEGURIDAD Y SEGURIDAD SOBRE LA INFORMACIÓN
}

\author{
SAFETY INFORMATION AND THE INFORMATION SURE
}

\section{AUTOR}

\section{Ángel Riesco García}

Inspector del Cuerpo Nacional de Policía (España)

a.riesco@hotmail.com

\section{RESUMEN}

El presente artículo plantea el sempiterno vital asunto de la seguridad y sus implicaciones en el ámbito de la información o comunicación. Es decir, la importancia del tratamiento informativo en la cobertura de asuntos sobre seguridad ciudadana, así como la responsabilidad que conlleva por su delicadeza y relevancia.

\section{PALABRAS CLAVE}

Seguridad- Información - Medios de comunicación

\section{ABSTRACT}

The present article raises the everlasting vital matter of the safety and his implications in the area of the information or communication. That is to say, the importance of the informative treatment in the coverage of matters on civil safety, as well as the responsibility that he carries for his sensitivity and relevancy. 


\section{KEY WORDS}

Security - Information - Mass media

\section{ÍNDICE}

1. Introducción

2. Seguridad

3. Información y seguridad

4. Conclusiones

\section{Introducción}

Puede ser de gran interés el análisis de cómo la generalidad de los ciudadanos llega a formar su particular visión, su vivencia exclusiva, su actitud respecto de un tema que atormenta a más de uno, la tan deseada seguridad. Igualmente, puede resultar aleccionador comprobar que lo que se cree, lo que se dice pensar sobre la seguridad, no se corresponde en absoluto con la realidad. Un porcentaje alto de la población mantiene creencias generalizadas, sufre una distorsión de la realidad, fruto de planteamientos simplistas, que es necesario desterrar por el bien de todos.

La seguridad tiene evidentemente dos parcelas fundamentales:

1. La seguridad físico-psíquica (frente a la violencia e intimidación).

2. La seguridad económica. 


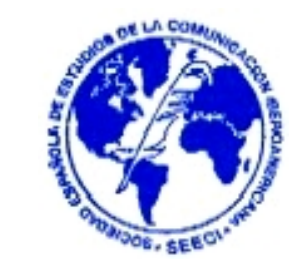

La seguridad emula los mecanismos que aseguran un buen funcionamiento de las cosas, que nos permiten aventurarnos sin demasiados riesgos, que nos protegen de peligros o daños. Es un valor siempre en alza, es un mito que todos queremos alcanzar, como si camináramos inexorablemente hacia una ideología de la seguridad, reflejo de la máxima aspiración social, al precio que sea.

Pocos son conscientes de que estamos condenados a no poder conseguir del todo esto que tanto deseamos, que la seguridad es, efectivamente, un mito no controlable por el hombre, o por lo menos en la medida deseada.

\section{Seguridad}

La seguridad puede representarse por dos planos móviles, llamados a cruzarse. Por un lado la persona desnuda, pero con ese deseo de eliminar el riesgo inherente que conlleva la vida, tan sólo con nacer. Por otro, la cancha o terreno de juego donde se desarrolla esa vida, el marco físico y social en donde cada uno está involucrado. Porque la cuestión es que la sociedad es desigual, conflictiva, y contradictoria; y que, en muchas ocasiones, la seguridad de unos se construye a base de la inseguridad de otros.

Sabemos que la seguridad es ante todo un sentimiento, una emoción que se siente 0 no, que se percibe o no, que se disfruta o no, y son muchos los factores que influyen para que una persona tenga o no esa sensación de seguridad o por el contrario de inseguridad. Los dato objetivos de la inseguridad se traducen en las cifras de delincuencia y los datos subjetivos se manifiestan a través de un crecimiento notable del temor. Famosos multimillonarios han tenido a su alcance todos los medios de protección y de seguridad que se pueden desear: sin embargo, no por ello han conseguido ese clima de seguridad que perseguían. 
Creo que no cabe duda de que nuestras expectativas respecto de la seguridad no son las adecuadas y ello se debe a que la información que recibimos no es la más idónea; a que por tanto la comunicación ha sido mal utilizada. Una parte importante de la población identifica la inseguridad con la falta de suficiente represión y prevención de los delitos, así como con el aumento real o supuesto de la criminalidad. En ocasiones, estas creencias llevan a planteamientos simplistas, poco rigurosos e incluso peligrosos. Detrás del aumento de la delincuencia puede haber todo un conglomerado de factores, pero la mayoría de las veces, detrás del miedo y la inseguridad hay una operación política encaminada a suscitar más demandas de control.

Existen dos grandes tipos de delincuencia:

1. Una que se podría considerar "tradicional", que percibimos más cerca de nosotros y que, por ello, contribuye directamente y en mayor medida a acrecentar nuestra sensación de seguridad. Son esos pequeños delitos que se comenten en la vía pública, donde nuestra seguridad física pues estar más amenazada.

2. No obstante, existe otro tipo de delincuencia mucho más inadvertida, más silenciosa, que aparece relacionada con estructuras políticas y económicas de poder, constituida por actividades socialmente mucho más graves $y$, sin embargo, de menor incidencia en el sentimiento de inseguridad.

La protección de las cosas materiales, de los bienes tangibles, de todo aquello que se puede ver y tocar, era la idea básica sobre la que descansaba, insisto, tradicionalmente, la seguridad. Si queríamos proteger un edificio lo rodeábamos de una valla infranqueable, incluso electrificada, podíamos añadir vigilantes armados, perros guardianes, detectores, cámaras de vídeo con infrarrojos, alarmas, etc. Pero, todo esto ha cambiado, ahora las cosas que no se ven y que no se pueden tocar 
necesitan también un creciente grado de protección. Entre estas "cosas" se encuentran:

- Las ideas.

- Los flujos electrónicos.

- La información.

- Etc ...

\section{Información y seguridad}

¿Estamos preparados para asumir esta evolución cultural tan repentina?

Normalmente, la mayoría de los ciudadanos llegan a alimentar su opinión sobre la seguridad a través, sobre todo, de las instituciones de transmisión cultural: familia, escuela, medios de comunicación, etc. Cuando empezamos a crecer empezamos a oír: "no hables con personas desconocidas"; en casa, nos recuerdan constantemente "no abras la puerta a nadie". En el colegio, también recibimos más información, nos llegan los comentarios del tipo de “ ¿sabes lo que le pasó a fulano...?. Más tarde, nos influyen los medios de comunicación con sus habituales y a menudo desproporcionados relatos de sucesos $\mathrm{y}$, entre ellos, las televisiones, con el afloramiento de programas, mitad misterio, mitad realidad, donde el dramatismo de novela barata llega a límites insospechados, produciendo alarma social.

Una gran parte de ciudadanos, lejos de la realidad criminal, no perciben como actividades que afecten a la convivencia, dentro de ese marco social al que aludíamos, cuestiones del tipo del "pirateo" informático, las copias indiscriminadas de vídeos, las fotocopias de libros, etc. No goza de la misma consideración una intromisión en nuestra casa que en nuestro ordenador. Otras conductas no nos resultan en absoluto tramposas, las percibimos fuera de nuestro contexto y no nos 
preocupan demasiado; por ejemplo, la economía sumergida, el fraude fiscal, los temas ecológicas, la delincuencia sin víctima, etc.

La comunicación es un instrumento de contacto, de convivencia, de interacción, de formación de opinión y actitudes, de influencia, etc., que puede utilizarse de una u otra forma, según el fin que se persiga. Aparte de sus acepciones habituales: impedir, estorbar, prepararse de antemano, etc., la prevención de delitos conlleva otro aspecto importante: el de "informar". Debe informarse de lo que sucede realmente, con sus ventajas e inconvenientes, porque siempre hay que asumir el riesgo de la realidad, de vivir. Pero, naturalmente, se deben contar las cosas de forma auténticamente real, sin añadir dramatismo, misterio y, por consiguiente, sin causar desproporcionada alarma social. Cuando los mismos medios de comunicación repiten una y otra vez, de una y otra forma, en una y otra sección o programa, quizá por falta de otros temas de interés, la noticia de un homicidio, se dispara un efecto multiplicador y da la sensación que los homicidios de aquel tipo son muy frecuentes.

Se debe informar sin incrementar el dramatismo, por supuesto sin intentar crear alarma social, sin inventar lo que no sabemos, sin rodear de misterio innecesario las noticias criminales. Los medios suelen informar sobre los hechos criminales y la forma de cometerse (el "modus operandi"), y esto tiene la ventaja de que, al conocer su desarrollo con detalle, podemos tomar una serie de precauciones para que no nos suceda a nosotros, pero puede tener el inconveniente de que surjan imitadores.

En cuanto al tema concreto de la victimización, ya el VIII Congreso de las Naciones Unidas sobre prevención del delito y tratamiento del delincuente, celebrado en La Habana (Cuba) del 27 de agosto al 7 de septiembre de 1990, estableció toda una serie de criterios y directrices. En lo que se refiere a publicidad y medios de información, consideró que dar publicidad a las necesidades de la víctima es un medio esencial para educar a los profesionales y al público en general, de forma que comprendan los efectos de la delincuencia. Sin embargo, hay que tener en cuenta 
que la publicidad detallada puede causar consternación suplementaria a las víctimas en determinados casos si, por ejemplo, se dan a conocer los nombres y direcciones de las mismas, lo que incluso puede exponerlas a una nueva victimización. Ciertos delitos causan tal aflicción a las víctimas que toda identificación de éstas en los medios de comunicación, especialmente en la televisión, tiene consecuencias graves. En lo que respecta a ciertos casos (por ejemplo, la violación) algunos países han promulgado leyes que prohíben nombrar a las víctimas. Es preferible y se debiera obtener siempre el consentimiento de la víctima antes de identificarla en los medios de comunicación. Es menester, obviamente, que los organismos de policía y justicia penal tengan sumo cuidado con la información que facilitan a los medios.

Otro aspecto informativo clave puede ser la identidad de los delincuentes. Aquí también los medios de comunicación deben ser cautos y prudentes. En primer lugar, la protección de los menores impide que su identidad aparezca en los medios, aunque hubieran cometido uno o varios delitos, pero, en segundo lugar, hay que pensar que no todas las personas que son detenidas por la presunta comisión de un ilícito penal son condenadas; muchas ce ellas son declaradas inocentes por un Tribunal o un Jurado, y el daño causado a su imagen sería irreparable. Tampoco se debe disculpar, cuando no ensalzar, la figura de los delincuentes y presentarlos ante nosotros o nuestros hijos como prácticamente un ejemplo a seguir; al menos, llegado el mismo caso.

Otro aspecto sobre el que se puede informar es sobre el trabajo policial. Debemos conocer realmente lo que hacen nuestras policías, qué persiguen, cómo lo hacen, ya que todos debemos ser un poco copartícipes de su trabajo, de acuerdo al modelo comunitarios. Una vez más, hay que cuidar de no exagerar las informaciones sobre investigaciones, ya que en ocasiones no son tan extraordinarias como se pretende. 
Entremos ahora a valorar una serie de instrumentos que pueden ser sumamente útiles para llegar a tener un conocimiento mucho más riguroso de la realidad criminal.

Desde el siglo XIX se utilizan las series estadísticas, ya sean policiales, judiciales 0 penitenciarias, como elementos para el conocimiento del crimen. Otros sistemas más modernos son los métodos de orden socio-criminológico y de orden económicocriminológico.

Entre los primeros, están las encuestas de autoconfesión ("Self report Surrey"), que han contribuido a desmontar la teoría de que el delito es un hecho minoritarios y a mostrar que, en contra de lo que se pensaba, éste se distribuye por toda la pirámide social.

Otro método socio-criminológico son las encuestas de victimización, que se pueden analizar desde dos puntos de vista muy distintos:

- Desde el hecho de ser víctima.

- Desde el miedo a serlo.

Se pregunta en primer lugar si la persona entrevistada ha sufrido algún percance con relación a su vivienda, trabajo, vehículo o sobre su seguridad personal, lo que hace que aflore la llamada "cifra negra" ("dark Lumber").

En cuanto a los métodos de orden económico-criminológico, se componen de estudios sobre el coste económico del delito, la correlación entre los diversos indicadores económicos y delito, el valor de lo sustraído, el valor de lo recuperado, la política de indemnizaciones a las víctimas, etc. 
Se obtiene de este trabajo sistemático gran cantidad de material informativo que, aunque no sea "noticia" (en el sentido de "anécdota"), puede generar un creciente interés en la sociedad, al igual que nos hemos habituado a la información sobre los índices y tendencias económicas.

\section{Conclusiones}

Antes de dar por terminada esta primera aproximación a este asunto, a modo de reflexión, podemos hacernos de nuevo las siguientes preguntas:

- Nuestras expectativas respecto a la seguridad ¿son las adecuadas?

- ¿Existe la seguridad total?

- La comunicación ¿juega un papel importante en nuestra seguridad?

- ¿Debe existir una especialización informativa en los aspectos relacionados con la seguridad (como existe en la economía), que no resida sólo en los reporteros de sucesos?

- ¿Contribuye la información actual a aumentar, o por el contrario, a disminuir el sentimiento de seguridad (y la seguridad misma)?

- ¿Percibimos de forma adecuada lo que sucede a nuestro alrededor y tiene relación con nuestra seguridad en diversos sentidos?

- ¿Es la "seguridad", en tanto o exista una información adecuada, una suma de componentes irracionales de fácil explotación? 


\section{Bibliografía}

FERNÁNDEZ PEDEMONTE, D. (2001): La violencia del relato. Discurso periodístico y casos policiales. La Crujía ediciones. Buenos Aires.

GOLBERT, L. y KESSLER, G. (2001): El crecimiento de la violencia urbana en la Argentina de los 90. Buenos Aires.

PEGORARO, J. (2000): Violencia delictiva, inseguridad urbana. La construcción social de la inseguridad ciudadana. Nueva Sociedad. Madrid.

REGUI LLA, R. (2000): La construcción social del miedo. Rotken. México.

WACQUANT, L. (2000): Las cárceles de la miseria. Manantial. Buenos Aires.

ZAFFARONI, E. R. (1989): En busca de las penas perdidas. Desligitimación y dogmática jurídico-penal. Ediar. Buenos Aires. 